\title{
Matrix metalloproteinases 15 and 19 are stromal regulators of colorectal cancer development from the early stages
}

\author{
PAOLA SENA $^{1^{*}}$, FRANCESCO MARIANI $^{2 *}$, LAURA MARZONA $^{1}$, MARTA BENINCASA $^{1}$, \\ MAURIZIO PONZ DE LEON ${ }^{2}$, CARLA PALUMBO $^{1}$ and LUCA RONCUCCI ${ }^{2}$ \\ Departments of ${ }^{1}$ Biomedical Sciences, Section of Human Morphology and ${ }^{2}$ Internal Medicine, \\ University of Modena and Reggio Emilia, I-41125 Modena, Italy
}

Received December 2, 2011; Accepted February 13, 2012

DOI: 10.3892/ijo.2012.1441

\begin{abstract}
Matrix metalloproteinases (MMPs) have been well characterized for their ability to degrade extracellular matrix proteins and, thus, they have been studied to elucidate their involvement in both tumor development and progression. In the present study, attention was focused on MMP-15 and MMP-19, two less known members of the MMP family. The expression profile of MMP-15 and -19 was assayed in samples of normal colorectal mucosa, microadenomas and cancer using confocal analysis, western blotting and quantitative reverse transcription polymerase chain reaction (qRT-PCR). Both qRT-PCR and western blotting showed that MMP-15 and MMP-19 appeared to be upregulated during colorectal tumorigenesis, with different expression patterns: MMP-15 expression level increases from normal mucosa to microadenomas, with a reduced level in cancer with respect to microadenomas; the semiquantitative immunofluorescence analysis showed a stromal localization of this protein in the early phases of neoplastic transformation. Increasing amount of MMP-19 mRNA and protein levels were observed in the progression of colonic lesions; MMP-19 staining increased in the normal mucosa-microadenoma-carcinoma sequence. Such different expression patterns, are probably due to the different roles played in colorectal tumorigenesis by these two molecules. Conflicting data on the role of these proteins in tumor progression have been reported, thus, an improved understanding of the biological roles of MMPs, in particular the lesser known members such as MMP-15 and 19, in colorectal cancer may lead to a re-evaluation of the use of MMP inhibitors and suggests the need of integrated translational studies on MMP expression patterns.
\end{abstract}

Correspondence to: Dr Luca Roncucci, Department of Internal Medicine, University of Modena and Reggio Emilia, Largo del Pozzo 71, I-41125 Modena, Italy

E-mail: luca.roncucci@unimore.it

*Contributed equally

Key words: matrix metalloproteinases, microadenomas, human colorectal carcinogenesis, qPCR, immunofluorescence

\section{Introduction}

Matrix metalloproteinases (MMPs) play a sophisticated role in cancer development due to their abilities to degrade various substrates. MMPs are generally classified as pro-angiogenic factors, since they can degrade the extracellular matrix molecules to facilitate tumor cell migration and invasion $(1,2)$. This concept of the role of MMPs in tumor invasion and metastasis is currently undergoing a major reappraisal most notably as a result of the apparent failure of several 'high-profile' clinical trials of MMP inhibitors in cancer (3).

MMP-15 is a recently discovered membrane-type MMP which was originally isolated from a human lung cDNA library, and it consists of a 76-kDa product with $73.9 \%$ overall similarity to MMP-14 $(4,5)$. Despite the high degree of structural similarity of these two MMPs, differences in substrate specificity $(6,7)$, as well as tissue or cellular localization, have been demonstrated (8). Although MMP-15 is reported to play a similar role to MMP-14 in cell invasion (9), its implication remains to be elucidated; the importance and role of MMP-14 in promoting tumor growth have been extensively investigated (10), in contrast to the role and function of MMP-15 which is just beginning to be addressed. It has been reported involved in the progression of various kinds of cancers, such as breast, cervical, and ovarian cancer (11-13). Recent reports indicate MMP-15 as an anti-apoptotic factor in cancer cells (14). Moreover, MMP-15 is also identified as prognostic predictor for disease-free survival in colorectal cancer (15).

Matrix metalloproteinase 19 (MMP-19) is a secreted protease, able to cleave various extracellular matrix (ECM) components and it is up-regulated under inflammatory conditions. MMP-19 was first isolated as an autoantigen, from the synovium of rheumatoid arthritis patients (16). The role of MMP-19 in promoting tumor invasion is as controversial as for all other MMPs. MMP-19 is up-regulated in astroglial tumor and melanoma $(17,18)$, and it is reported to increase human keratinocyte cell proliferation, migration and adhesion on type I collagen through the IGF-signalling pathway (19). These findings suggest that MMP-19 may play a role during malignant transformation. On the other hand, some studies reported that MMP-19 is down-regulated or even lost during neoplastic progression in breast and skin (20-22). Evidence is emerging for important biological roles of MMPs including 
apoptosis, cell proliferation and cell differentiation (14). Much of the evidence linking MMPs to wider biological roles in both tumor development and progression are being provided by the study of colorectal cancer, since it represents a welldefined and extensively studied model of tumorigenesis as well as being among the most common malignancies worldwide and one of the leading causes of cancer-related death. In this respect, the present study focused on the analysis of the expression of MMPs 15 and 19, from the earliest stages of neoplastic transformation to cancer. The results clearly show that the proteins investigated exhibit different behavior during tumor progression.

\section{Materials and methods}

Study population. Samples $(\mathrm{n}=56)$ of normal colorectal mucosa (NM) were collected from 14 patients during colonoscopy, at least 3 samples for each patient. One sample for each patient was fixed in formalin and embedded in paraffin for histology and RNA extraction; the others were frozen at $-80^{\circ} \mathrm{C}$. All patients had normal colonoscopy. Thirty microadenomas (MA) were also identified in 16 patients, and removed after operation for colorectal cancer on surgical specimens, after staining of the mucosa with a $0.1 \%$ methylene-blue solution in saline, and observation under a dissecting microscope (23). All microadenomas were defined as dysplastic aberrant crypt foci at histology and, indeed, they could be referred to as microadenomas. Ten MA were fixed in formalin and embedded in paraffin, the others were frozen at $-80^{\circ} \mathrm{C}$. Finally, 33 samples of colorectal cancer (CRC) were collected from 11 patients operated on for cancer, fixed in formalin or frozen at $-80^{\circ} \mathrm{C}$, as described above.

All the patients enrolled in this study, who underwent colonoscopy or surgical resection for colorectal cancer at the University Hospital of Modena, gave their informed written consent to the study protocol, which was approved by the local Ethics Committee.

Immunofluorescence confocal microscopy. Immunofluorescence analysis was carried out to evaluate the expression of MMP-15 and MMP-19 proteins. Multiple labelling of the samples was carried out to analyze the co-localization of MMP-15/MMP-19 with vimentin, myeloperoxidase, CD3 and CD20, in order to determine their possible interaction. Samples of NM, MA, and CRC were fixed in $4 \%$ paraformaldehyde in PBS, cryoprotected in $15 \%$ sucrose in PBS, and frozen in isopentane cooled in liquid nitrogen. Horizontal cryosections of the samples were cut $(10-\mu \mathrm{m}$ thick), and hematoxylin and eosin staining was performed on sections to control tissue integrity. After a treatment with 3\% BSA in PBS for 30 min at room temperature, the cryostatic sections were incubated with the primary antibodies (rabbit anti-MMP-15, rabbit anti-MMP-19, Novus Biologicals; mouse anti-vimentin, mouse anti-myeloperoxidase, mouse anti-CD3, mouse anti-CD20 DakoCytomation); diluted 1:25 in PBS containing 3\% BSA for $1 \mathrm{~h}$ at room temperature. After washing in PBS, the samples were incubated for $1 \mathrm{~h}$ at room temperature with the secondary antibodies diluted 1:20 in PBS containing 3\% BSA (sheep anti-mouse FITC conjugated, goat anti-rabbit $\mathrm{Cy} 3$ conjugated, goat anti-rabbit TRITC-conjugated; Sigma). After washing in PBS and in $\mathrm{H}_{2} \mathrm{O}$, the samples were counterstained with $1 \mu \mathrm{g} / \mathrm{ml}$ DAPI in $\mathrm{H}_{2} \mathrm{O}$ and then mounted with anti-fading medium (0.21 M DABCO and $90 \%$ glycerol in 0.02 M Tris, $\mathrm{pH}$ 8.0). Negative control samples were not incubated with the primary antibody. The confocal imaging was performed on a Leica TCS SP2 AOBS confocal laser scanning microscope. For DAPI and Cy3 double detection, samples were sequentially excited with the $405-\mathrm{nm} / 25-\mathrm{mW}$ line of a blue diode laser and the 543-nm/1.2-mW line of a HeNe laser. The emission signals from DAPI and Cy3 were detected by two photomultiplier tubes. The samples, processed for triple fluorescence (DAPI, FITC and Cy3), were sequentially excited with the 405-nm/25-mW lines of a blue diode laser, the 488-nm/20-mW lines of the Argon laser and the 543-nm/1.2-mW lines of a HeNe laser.

Excitation and detection of the samples were carried out in sequential mode to avoid overlapping of signals. Sections were scanned with laser intensity, confocal aperture, gain and blacklevel setting kept constant for all samples. Optical sections were obtained at increments of $0.3 \mu \mathrm{m}$ in the $\mathrm{z}$-axis and were digitized with a scanning mode format of $512 \times 512$ or $1024 \times 1024$ pixels and 256 grey levels. The confocal serial sections were processed with the Leica LCS software to obtain three-dimensional projections. Image rendering was performed by adobe Photoshop software.

Evaluation of MMP-15 and MMP-19 immunofluorescence. The evaluation was carried out in a subset of samples, 6 for NM, 6 for MA, and 6 for CRC, randomly selected. The original green fluorescent confocal images were converted to greyscale and median filtering was performed. An intensity value ranging from 0 (black) to 255 (white) was assigned to each pixel. Background fluorescence was subtracted and the immunofluorescence intensity (IF) was calculated as the average for each selected area.

To quantify MMP-15 and -19 expression, all blocks were sectioned exhaustively and 3-4 slides were examined at magnification $\mathrm{x} 40$ for each patient. In each section almost 60 crypts were present. Starting randomly, 3 fields of vision for each section were used for sampling all crypts within the unbiased sampling frame.

The fluorescence intensity at the selected areas, linearly correlated with the number of pixels, was quantitatively analysed using the standard imaging analysis software of the NIS-Elements system. To each sample was assigned a code number and the score, referred to as immunofluorescence intensity score (IFIS), determined by an observer who was blind to tissue groups during analysis (23).

For each patient, 3-4 slides were examined at magnification $\mathrm{x} 40$ in order to identify the type/amount of stromal cells in which the co-localization of each MMP (15 or 19) was present. The correspondence between co-localization and cellular type was the following: MMP-15/19 with vimentin, identifies fibroblast-like cells; MMP-15/19 with myeloperoxidase, identifies neutrophils and macrophages; MMP-15/19 with CD3 identifies T- lymphocytes; MMP-15/19 with CD20 identifies B-lymphocytes. With respect to a total amount of $200 \mathrm{MMP}-$ positive stromal cells identifiable in each slide, the number of cells presenting merge signal was recorded.

This type of histomorphometrical evaluation (cell number, 200) is the best solution to obtain a sampling independent from the dimensions of the histological section. To each sample was assigned a code number and the score was determined by an observer who was blind to tissue groups during analysis. 

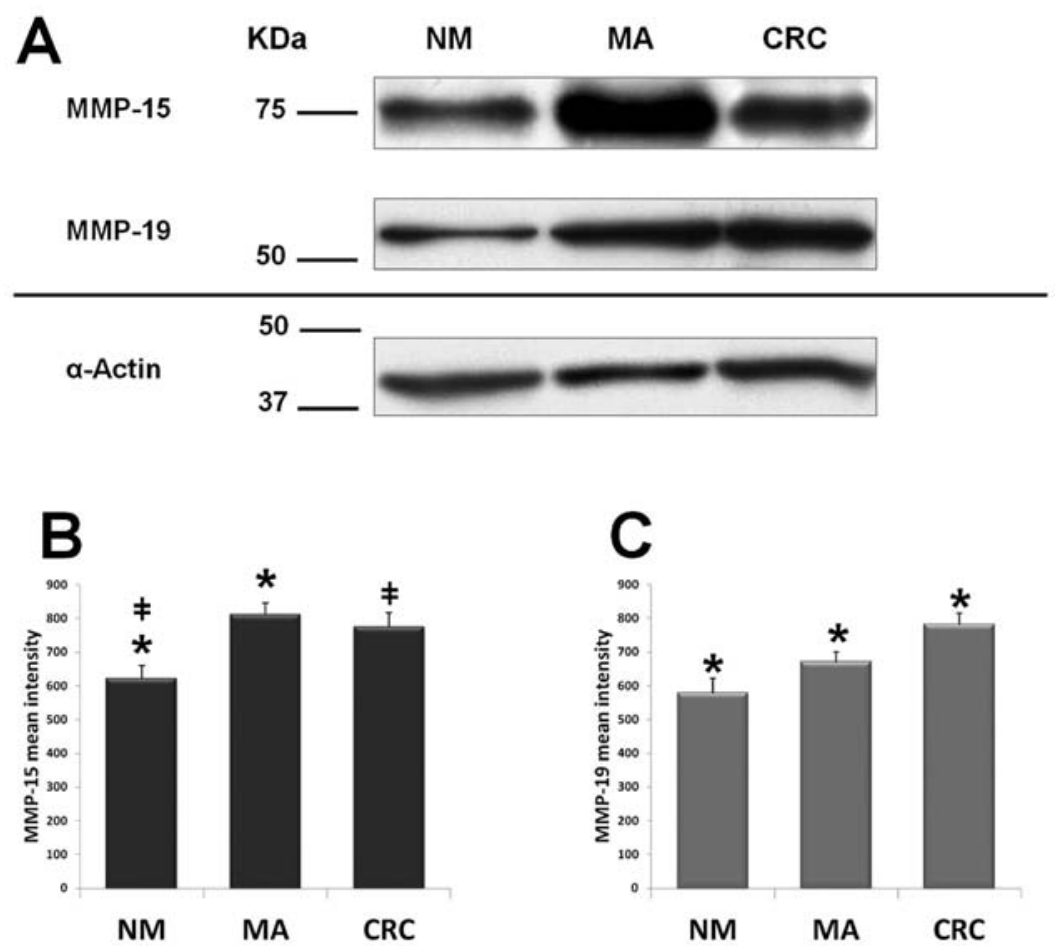

Figure 1. (A) Western blotting of metalloproteinases 15 and 19 (MMP-15 and -19) in human normal colorectal mucosa (NM), microadenomas (MA), colorectal carcinomas (CRC). (B) Mean densitometric data of MMP-15 protein expression level. (C) Mean densitometric data of MMP-19 protein expression level (p<0.05 for groups with the same symbol).

Western blot analysis. Whole cell lysates were obtained from the samples of NM, MA, and CRC, extracted with hypotonic buffer (50 mM Tris- $\mathrm{Cl}, \mathrm{pH} 7.8$, containing $1 \%$ Nonidet P40, $140 \mathrm{mM}$ $\mathrm{NaCl}, 0.1 \%$ SDS, $0.1 \%$ Na deoxycholate, $1 \mathrm{mM} \mathrm{Na}_{3} \mathrm{VO}_{4}$, and freshly added protease inhibitor cocktail). Lysates were then cleared by centrifugation for $15 \mathrm{~min}$ in a refrigerated centrifuge, at maximal speed, and immediately boiled in SDS sample buffer. Protein extract $(40 \mu \mathrm{g})$ from each sample (NM, MA, and CRC) was electrophoresed on SDS-PAGE and transferred to nitrocellulose membranes. The protocols of the Western blotting were performed as previously described (23). The membranes were blocked with $3 \%$ dry milk and 2\% BSA in PBS-T and was incubated with the following antibodies, diluted 1:1000 overnight at $4^{\circ} \mathrm{C}$ under agitation: rabbit anti-MMP-15, rabbit anti-MMP-19, Novus Biologicals. After washing, the membranes were incubated with secondary HPR-conjugated goat anti-rabbit IgG antibody $(1: 10,000)$ for $30 \mathrm{~min}$ at room temperature. Immunoreactive proteins were detected with ECL (Amersham). Furthermore, the membranes were stripped and incubated with anti-mouse $\alpha$-actin (Sigma) to control and correct for loading error. Densitometry analysis was performed using a Kodak (Rochester, NY) Image Station 440cf system.

RNA isolation and $q R T-P C R$. mRNA was isolated from formalin-fixed, paraffin-embedded samples of all cases. Total RNA was extracted from $10-\mu \mathrm{m}$ sections, using High Pure RNA Paraffin Kit (Roche Diagnostics S.p.A., Milan, Italy). RNA content was determined by spectrophotometry and firststrand cDNA was synthesized from $2 \mu \mathrm{g}$ of total RNA with Superscript VILO cDNA synthesis kit (Invitrogen S.r.l., Milan, Italy) according to the manufacturer's instructions.
Real-time PCR was done on the iCycler iQReal-Time Detection System (Bio-Rad) using Go-taq qPCR Master Mix (Promega, Milan, Italy) according to detailed manufacturer's protocols. To confirm amplification of a specific product, melting curve analysis was performed and PCR products were directly visualized on $2 \%$ low-melting agarose gels.

The MMP-15 and MMP-19 amplification results were normalized to the corresponding GAPDH results. Data were expressed as fold induction in pathological tissue compared to normal mucosa ( $\Delta \mathrm{Ct}$ method using a reference gene or the Livak method) (24).

Specific primer pairs were as follows: MMP-15, forward 5'-ccgccctcattatttatgtcc-3' and reverse 5'-attctaaacacctgcccc-3'; MMP-19, forward 5'-tgctttcaagggggactatg-3' and reverse 5'-agaca gcagcatccaggttt-3'; GAPDH, forward 5'-agccacatcgctcagacac-3' and reverse 5'-gcccaatacgaccaaatcc-3' (used as normalized). The cycling conditions were established as follows: single cycle at $95^{\circ} \mathrm{C}$ for $2 \mathrm{~min}, 40$ cycles at $95^{\circ} \mathrm{C}$ for $15 \mathrm{sec}$, and at $60^{\circ} \mathrm{C}$ for $60 \mathrm{sec}$.

Statistical analysis. All quantitative data for NM, MA, and CRC are reported as mean \pm SD or mean \pm SEM. The difference in MMP-15 and MMP-19 average expression in the different groups of colorectal lesions, was tested for statistical significance using Kruskal-Wallis analysis, followed by Student-Newman-Keuls tests. $\mathrm{p}<0.05$ was chosen to indicate a statistical significant difference.

\section{Results}

MMP-15 quantification in the early steps of colorectal carcinogenesis. Western blotting of cell lysates in analysing the 

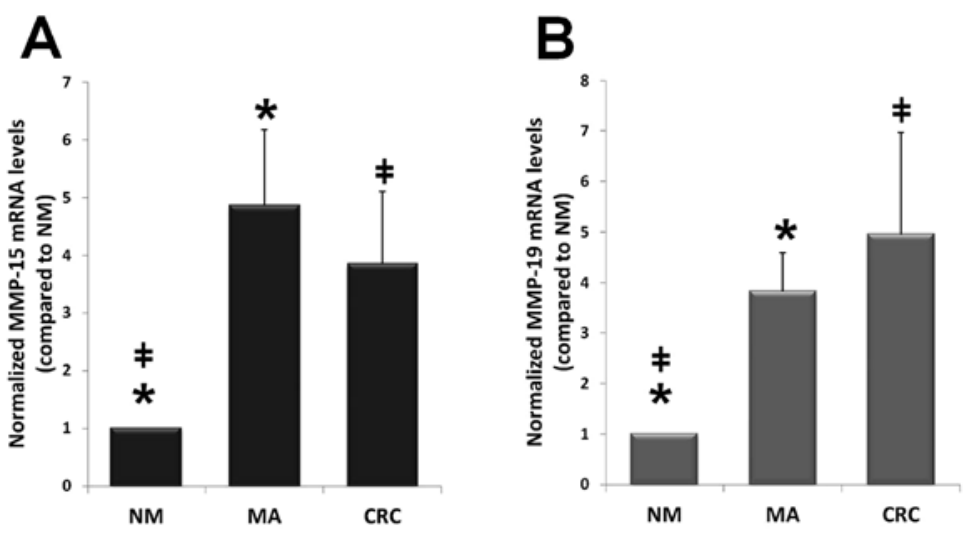

Figure 2. (A) qRT-PCR results for MMP-15 expressed as fold change with respect to NM ( $p<0.05$ for groups with the same symbol). (B) $q R T-P C R$ results for MMP-19 expressed as fold change with respect to NM ( $\mathrm{p}<0.05$ for groups with the same symbol).

Table I. Immunofluorescence intensity score (IFIS), and mRNA quantification of metalloproteinases 15 and 19 (MMP-15 and MMP-19) in human normal colorectal mucosa (NM), microadenomas (MA) and colorectal carcinomas (CRC).

\begin{tabular}{lcccc}
\hline & \multicolumn{2}{c}{ MMP-15 } & & MMP-19 \\
\cline { 2 - 4 } & IFIS (mean \pm SD) & $\begin{array}{c}\text { mRNA fold change } \\
(\text { mean } \pm \text { SEM) }\end{array}$ & & $\begin{array}{c}\text { IFIS (mean } \pm \text { SD) } \\
\text { mRNA fold change } \\
(\text { mean } \pm \text { SEM) }\end{array}$ \\
\hline NM & $30.7 \pm 9.0^{\mathrm{a}}$ & 1.0 & $36.1 \pm 5.0^{\mathrm{a}}$ & 1.0 \\
MA & $96.7 \pm 9.9^{\mathrm{a}}$ & $4.9 \pm 1.3^{\mathrm{b}}$ & $74.6 \pm 4.0^{\mathrm{a}}$ & $3.8 \pm 0.8^{\mathrm{b}}$ \\
CRC & $79.2 \pm 5.4^{\mathrm{a}}$ & $3.8 \pm 1.2^{\mathrm{b}}$ & $107.0 \pm 7.8^{\mathrm{a}}$ & $4.9 \pm 2.0^{\mathrm{b}}$ \\
\hline
\end{tabular}

${ }^{\mathrm{a}} \mathrm{p}<0.05$ between all group pairs of samples. ${ }^{\mathrm{b}} \mathrm{p}<0.05$ vs $\mathrm{NM}$.

protein profile of MMP-15 in normal mucosa (NM), microadenomas (MA) and colorectal cancer (CRC), clearly showed one single weak band at the expected molecular weight (76 kDa) in NM, a very intense band in MA, and a less intense band in CRC samples (Fig. 1A).

Densitometric analysis and normalization (with equal amounts of protein loading) of the immunoreactivity signal from protein extracts of NM, MA and CRC showed that the level of MMP-15 protein have an upward trend from NM to MA, with statistical significance of the different expression. Protein levels slightly decreased from MA to CRC (Fig. 1B).

The qRT-PCR analysis, to investigate the MMP-15 transcriptional activation, was first performed on fresh tissue, and then on formalin-fixed, paraffin-embedded samples, giving comparable yields. Fig. 2A, and Table I summarize the expression levels of MMP-15 in MA and CRC, versus NM. The amount of MMP-15 mRNA was higher in MA and in CRC compared to NM. Data of protein expression and mRNA quantification were strictly correlated: they showed a peak of both MMP-15 protein and mRNA in the early dysplastic lesions (MA), suggesting that MMP-15 activation is essential in the early steps of CRC development.

Stromal localization of MMP-15 in colorectal carcinogenesis. Confocal analysis of MMP-15 in samples of NM, MA, and CRC allowed to define both the distribution pattern and the quantification of this protein. The staining patterns of MMP-15 varied from a diffuse mode to a granular one in the cytoplasm of stromal cells, appearing consistent and unchanged in the same samples, without considerable variation in intensity and localization from cell to cell.

All samples of NM were not marked at the epithelial level, although a few scattered areas in the adjacent stroma showed a moderate MMP-reactivity (Fig. 3A). In MA intense staining was evident in stromal cells surrounding colonic crypts, whereas epithelial cells did not show any expression (Fg. 3B). In CRC samples the overall intensity of MMP-15 staining was more evident in comparison with NM, although it was decreased compared with MA (Fig. 3C). Cancer epithelial cells showed undetectable MMP-15 reactivity.

These features were confirmed by the measurement of the MMP-15 expression levels (Table I). In order to clarify the role of the stromal compartment in the synthesis of MMP-15, we tried to identify the type of stromal cells mostly involved in this process. For this purpose, double immunofluorescence analyses were performed with the MMP-15 antibody together with several antibodies specific for different stromal cells, i.e., anti-vimentin, specific for fibroblast-like cells, anti-myeloperoxidase, specific for neutrophils and macrophages, anti-CD3 and anti-CD20, which recognise $\mathrm{T}$ and $\mathrm{B}$ lymphocytes, respectively. Interestingly, the staining profiles observed showed the greatest amount of MMP-15 positive cells, $70 \%$, in fibroblast-like cells and $30 \%$ neutrophils/macrophages, as revealed by coimmunostaining with MMP-15 and the antibodies specific for these different cell types (Fig. 3G-I). Very few MMP-15-positive lymphocytes were observed in the tumor stroma. This expres- 

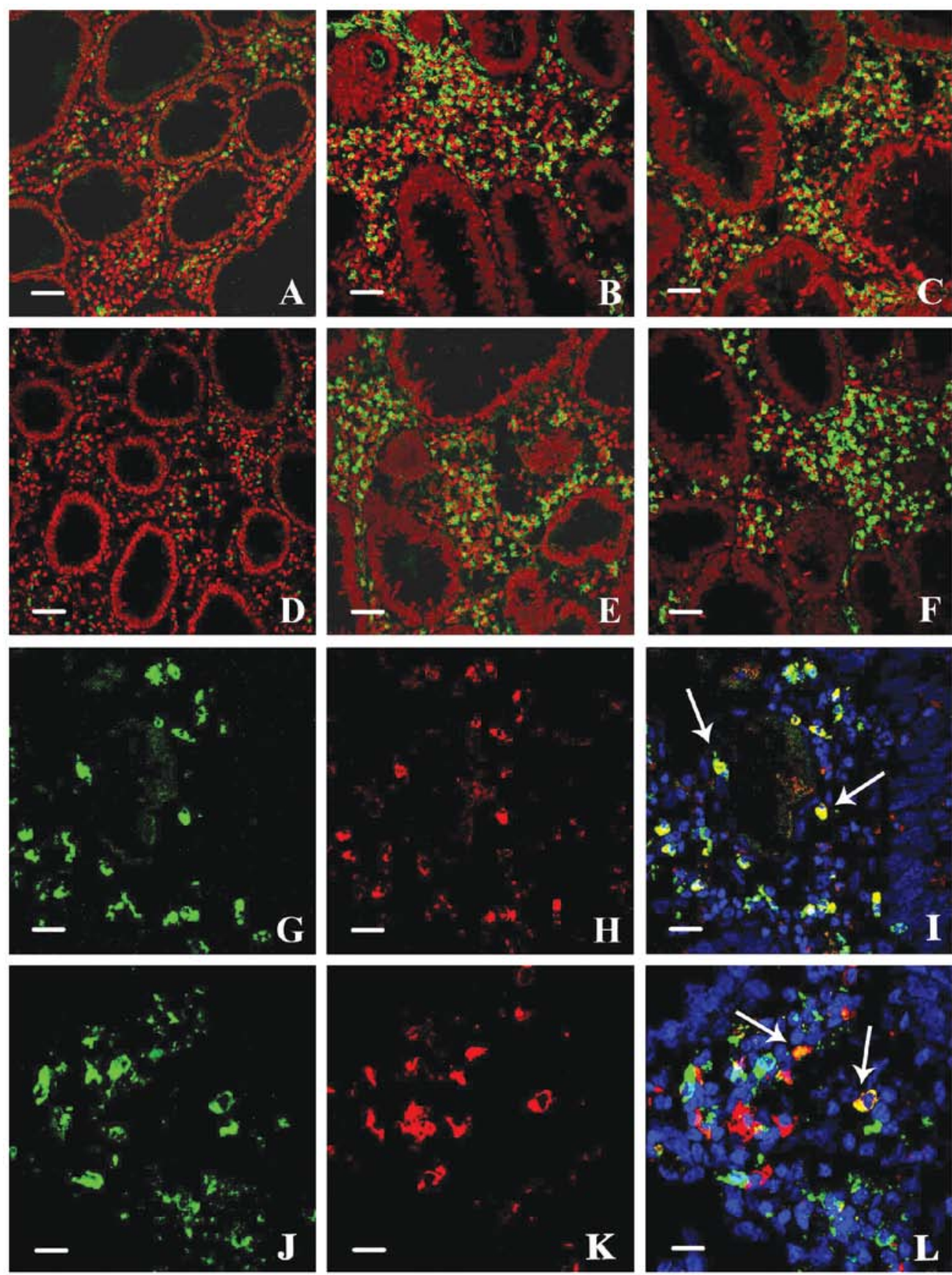

Figure 3. Confocal analysis of colonic mucosa cryosections labelled by DAPI (A-F, red; I and L, blue), MMP-15 (green), MMP-19 (green); and vimentin (H, I, K and $\mathrm{L}$, red). (A-C) The double immunolabelling performed by DAPI and MMP-15 shows an increased expression of the MMP-15 protein from normal mucosa (A) to microadenomas (B) which tends to decrease in carcinomas (C). (D-F) The image obtained by the merge of DAPI and MMP-19 shows a marked increase of staining in the sequence normal mucosa (D) -microadenoma (E) -carcinoma (F). (G-I) Samples of carcinoma stained with MMP-15, vimentin and DAPI. In the stromal compartment is evident the presence of many labeled cells with MMP-15 and vimentin, whose co-immunostaining (yellow) corresponds to 70\% (arrows). (J-L) Samples of carcinoma stained with MMP-19, vimentin and DAPI. Co-localization (yellow) of MMP-19 and vimentin in stroma cells point out the presence of fibroblast-like cells producing MMP-19 (arrows). Scale bars, 30 (A and D), 50 (B, C, E and F), 20 (G-I) and $15 \mu \mathrm{m}(\mathrm{J}-\mathrm{L}$ ).

sion profile was more evident in MA and CRC samples, whereas in NM it was difficult to identify the different cell types.

MMP-19 gradually increased from normal colorectal mucosa to microadenoma and carcinoma. The Western blotting profile of MMP-19 is shown in Fig. 1A. It was different from the MMP-15 one. MMP-19, as the $58 \mathrm{kDa}$ band, was more evident in CRC with respect to MA, but it was very slight in NM. The densitometric analysis (Fig. 1C), consistently showed that the amounts of
MMP-19 proteins increased significantly in the progression from NM to CRC. Formalin-fixed paraffin-embedded samples gave good results also for MMP-19 mRNA. The qRT-PCR expression data confirmed a steady increase of MMP-19 mRNA levels from NM to CRC (Fig. 2B).

MMP-19 immunofluorescence in stromal and epithelial cells. The expression pattern of MMP-19, evaluated in the stromal compartments was different from that observed for MMP-15; 
the samples of NM showed a weak staining, whereas the protein expression levels tended to increase from MA to CRC (Fig. 3D-F). In all samples, MMP-19 positive cells filled the lamina propria surrounding the colonic crypts (Fig. 3F).

The semiquantitative analysis of the IFIS score of MMP-19 showed a higher staining intensity in MA with respect to NM, further increased in CRC (Table I). Double immunofluorescence analysis aimed at identifying the type of stromal cells mainly involved in this process, showed a pattern similar to that observed for MMP-15, with a prevalence of fibroblast-like MMP-19 positive cells (Fig. 3J-L). Only a small fraction of the total population of myeloperoxidase positive cells showed a coimmunostaining with MMP-19 antibody.

\section{Discussion}

In the present study attention was focused on the evaluation of the expression of the MMP-15 and MMP-19, the less investigated members of the family of metalloproteases, during the neoplastic progression in human intestinal mucosa. Our immunofluorescence analysis showed that MMP-15 is expressed in normal mucosa, although at low levels, whereas it increases in microadenomas; such expression slightly decreased in carcinomas. These results were confirmed by western blotting and qRT-PCR data, with slight differences due to the various techniques used.

MMP-15 is a recently discovered membrane-type MMP (4); among the six membrane-type MMPs, only MMP-14 has been widely investigated and it is thought to play a crucial role in cellular invasion and proliferation (25). Thus, although MMP-15 is supposed to play a similar role as MMP-14 in cell invasion (9), less attention has been paid to MMP-15 and its role remains to be elucidated. The leading current hypothesis is that MMP-15 is involved in cancer progression, as inferable from the reports on the association of MMP-15 with various type of cancer (11-13). Furthermore, MMP-15 protein has been investigated and proposed as a possible prognostic factor in human colorectal carcinoma (15). Interestingly, our results indicate that the MMP-15 protein is highly expressed in the early stages of colorectal carcinogenesis. Indeed, it is now becoming evident that specific MMPs play a distinct roles in the early stages of colorectal tumorigenesis. Most of the evidence on this topic has accumulated for MMP-7 that could be considered as an oncogene; in fact, colorectal tumorigenesis is suppressed in mice lacking MMP-7 (26) and such MMP was showed to be involved in the initial steps of tumor development (27). Abraham et al (14) validated the anti-apoptotic activity of MMP-15 and hypothesized that this protease connects metastasis and apoptosis resistance by an unknown regulatory mechanism. Furthermore, they suggested that cancer characteristics such as metastatic potential, which are thought as late events in cancer progression, could be present early by the selection for an anti-apoptotic phenotype. This hypothesis is in line with the data of our previous work (23), where we demonstrated that apoptosis decreases significantly in microadenomas compared to normal colonic mucosa; the fact that apoptosis decreases is crucial in the early stages of neoplastic transformation, while apoptosis decrement, not drastic as in previous stages, may be less relevant for cancer progression.

In this study we also analyzed for the first time the behavior of the MMP-19 protein during the major milestones of the transformation from normal mucosa to colorectal carcinoma. We reported that MMP-19 is expressed in normal mucosa at low levels and it is up-regulated during neoplastic progression. The increased expression of MMP-19 observed during the transition from microadenomas to cancer is confirmed by both the semiquantitative analysis of fluorescence, and western blotting profile as well as the data obtained by the qRT-PCR method. These findings strongly suggest that MMP-19 may play a role during malignant transformation. However, some other works reported conflicting data about the role played by MMP-19 in cancer: for example, MMP-19 is up-regulated in astroglial tumor and melanoma $(17,18)$, and it is down-regulated or even lost during neoplastic progression in breast and skin $(20,21)$.

Our observations consistently showed that the MMP-19 protein is expressed only in the stromal compartment of the normal mucosa and its presence reaches the maximum levels in the tumor samples; all our qualitative and quantitative data (immunofluorescence, western blotting and qRT-PCR on mRNA) are consistent with this finding. On this consistent basis, the authors underlined the importance of MMP-19 mostly in the stromal ambit during tumorigenesis, although Bister et al (22) showed that MMP-19 is downregulated in the epithelium of invasive colon cancer islands failing, indeed, in showing quantitative data.

Another topic of the present study was the identification of the types of stromal cells producing MMP-15 and -19 proteins. Both matrix metalloproteinases (15 and 19) were expressed by stromal fibroblasts in high percentage and by macrophages in smaller amounts, which is in accordance with previous data on cell types expressing MMP-19 $(28,29)$. Several studies using mouse models of genetically altered fibroblasts demonstrated a direct involvement of resident fibroblasts in the onset of cancer (30); moreover, both genetic and cell-biology studies indicate that tumor growth is not just determined by malignant cancer cells themselves, but also by the tumor stroma (31). There is also compelling evidence of the importance of the relationship between stromal cells and colorectal carcinoma cells in promoting MMP production either in tumor cells or in stromal cells $(32,33)$. Some authors suggested the existence of a mechanism whereby invasive tumor cells secrete soluble factors which induce stromal cells (most probably fibroblasts) to produce MMPs (34), although several factors indicate that distinct mechanisms have evolved for the control of individual MMPs during tumor-host interaction.

In conclusion, in this study, for the first time, different behaviors of MMP-15 and -19 in human colorectal cancer development have been outlined; MMP-15 appears to be mainly active in the early stages of carcinogenesis performing a putative anti-apoptotic role, MMP-19 seems to be involved in tumor progression, as its expression increases significantly during neoplastic transformation.

The conventional view of MMPs as matrix-degrading enzymes, giving to these proteins a central role in tumor invasion simply as a consequence of their activity, is now being challenged by new concepts of the roles of MMPs in tumor biology. Two are the key emerging concepts for the MMPs as a multifunctional group of molecules with complex biological actions: firstly, individual MMPs have a different role in the early stages of tumor development; secondly, the identification of an expanding range of MMP substrates which are non-matrix proteins, provides the basis for considering the burgeoning 
range of biological functions attributed to MMPs, in particular those functions such as cell proliferation and cell death, crucial for tumor development and progression. The improved understanding of the biological roles of all the members of MMP family in colorectal cancer development should lead to a re-evaluation of the use of MMP inhibitors and highlights the importance of studying the MMPs in an integrated translational manner.

\section{Acknowledgements}

This work was supported by grants from the Italian Ministry of Education (grants COFIN), the Italian Association for Research on Cancer (AIRC), the Lega Italiana per la Lotta contro i Tumori (LILT), the Region Emilia-Romagna, and the Fondazione Umberto Veronesi (which supported the grant for Dr F. Mariani). For this study the confocal microscope Leica TCS SP2 of the C.I.G.S. (Centro Interdipartimentale grandi strumenti) of the University of Modena and Reggio Emilia was used.

\section{References}

1. Kalebic T, Garbisa S, Glaser B and Lotta LA: Basement membrane collagen: degradation by migrating endothelial cells. Science 221 : 281-283, 1983.

2. Sato H, Takino T, Okada Y, Cao J, Shinagawa A, Yamamoto E and Seiki M: Matrix metalloproteinase expressed on the surface of invasive tumour cells. Nature 370: 61-65, 1994.

3. Coussens LM, Fingleton B and Matrisian LM: Matrix metalloproteinase inhibitors and cancer: trials and tribulations. Science 29: 2387-2392, 2002.

4. Takino T, Sato H, Shinagawa A and Seiki M: Identification of the second membrane-type matrix metalloproteinase (MT-MMP-2) gene from a human placenta cDNA library. MT-MMPs form a unique membrane-type subclass in the MMP family. J Biol Chem 29: 23013-23020, 1995.

5. Sato H: Membrane-type matrix metalloproteinases (MT-MMPs) in tumour metastasis. J Biochem 119: 209-215, 1996.

6. d'Ortho MP, Will H, Atkinson S, Butler G, Messent A, Gavrilovic J, Smith B, Timpl R, Zardi L and Murphy G: Membrane-type matrix metalloproteinases 1 and 2 exhibit broad-spectrum proteolytic capacities comparable to many matrix metalloproteinases. Eur J Biochem 250: 751-757, 1997.

7. English WR, Puente XS, Freije JM, Knauper V, Amour A, Merryweather A, Lopez-Otin C and Murphy G: Membrane type 4 matrix metalloproteinase (MMP17) has tumour necrosis factoralpha convertase activity but does not activate pro-MMP2. J Biol Chem 275: 14046-14055, 2000.

8. Szabova L, Yamada SS, Birkedal-Hansen H and Holmbeck K Expression pattern of four membrane-type matrix metalloproteinases in the normal and diseased mouse mammary gland. J Cell Physiol 205: 123-132, 2005.

9. Hotary K, Allen E, Punturieri A, Yana I and Weiss SJ: Regulation of cell invasion and morphogenesis in a three-dimensional type I collagen matrix by membrane-type matrix metalloproteinases 1 , 2, and 3. J Cell Biol 149: 1309-1323, 2000.

10. Holmbeck K, Bianco P, Yamada S and Birkedal-Hansen H: MT1-MMP: A tethered collagenase. J Cell Physiol 200: 11-19, 2004.

11. Ueno H, Nakamura H, Inoue M, Imai K, Noguchi M, Sato H, Seiki $M$ and Okada Y: Expression and tissue localization of membrane-types 1,2, and 3 matrix metalloproteinases in human invasive breast carcinomas. Cancer Res 57: 2055-2060, 1997.

12. Davidson B, Goldberg I, Berner A, Nesland JM, Givant-Horwitz V, Bryne M, Risberg B, Kristensen GB, Tropé CG, Kopolovic J and Reich R: Expression of membrane-type 1, 2, and 3 matrix metalloproteinases messenger RNA in ovarian carcinoma cells in serous effusions. Am J Clin Pathol 115: 517-524, 2001.

13. Ito E, Yana I, Fujita C, Irifune A, Takeda M, Madachi A, Mori S, Hamada Y, Kawaguchi N and Matsuura N: The role of MT2-MMP in cancer progression. Biochem Biophys Res Commun 393: $222-227,2010$
14. Abraham R, Schafer J, Rothe M, Bange J, Knyazev P and Ullrich A: Identification of MMP-15 as an anti-apoptotic factor in cancer cells. J Biol Chem 280: 34123-34132, 2005.

15. Asano T, Tada M, Cheng S, Takemoto N, Kuramae T, Abe M, Takahashi O, Miyamoto M, Hamada J, Moriuchi T and Kondo S: Prognostic values of matrix metalloproteinase family expression in human colorectal carcinoma. J Surg Res 146: 32-42, 2008.

16. Sedlacek R, Mauch S, Kolb B, Schätzlein C, Eibel H, Peter HH, Schmitt J and Krawinkel U: Matrix metalloproteinase MMP-19 (RASI-1) is expressed on the surface of activated peripheral blood mononuclear cells and is detected as an autoantigen in rheumatoid arthritis. Immunobiology 198: 408-423, 1998.

17. Lettau I, Hattermann K, Held-Feindt J, Brauer R, Sedlacek R and Mentlein R: Matrix metalloproteinase-19 is highly expressed in astroglial tumours and promotes invasion of glioma cells. Neuropathol Exp Neurol 69: 215-223, 2010.

18. Müller M, Beck IM, Gadesmann J, Karschuk N, Paschen A, Proksch E, Djonov V, Reiss K and Sedlacek R: MP19 is upregulated during melanoma progression and increases invasion of melanoma cells. Mod Pathol 23: 511-521, 2010.

19. Sadowski T, Dietrich S, Koschinsky F and Sedlacek R: Matrix metalloproteinase 19 regulates insulin-like growth factor-mediated proliferation, migration, and adhesion in human keratinocytes through proteolysis of insulin-like growth factor binding protein-3. Mol Biol Cell 14: 4569-4580, 2003.

20. Djonov V, Högger K, Sedlacek R, Laissue J and Draeger A: MMP-19: cellular localization of a novel metalloproteinase within normal breast tissue and mammary gland tumours. J Pathol 195: 147-155, 2001.

21. Impola U, Toriseva M, Suomela S, Jeskanen L, Hieta N, Jahkola T, Grenman R, Kähäri VM and Saarialho-Kere U: Matrix metalloproteinase-19 is expressed by proliferating epithelium but disappears with neoplastic dedifferentiation. Int J Cancer 103: 709-716, 2003.

22. Bister VO, Salmela MT, Karjalainen-Lindsberg ML, Uria J, Lohi J, Puolakkainen P, Lopez-Otin C and Saarialho-Kere U: Differential expression of three matrix metalloproteinases, MMP-19, MMP-26, and MMP-28, in normal and inflamed intestine and colon cancer. Dig Dis Sci 49: 653-661, 2004.

23. Sena P, Roncucci L, Marzona L, Mariani F, Maffei S, Manenti A and De Pol A: Altered expression of apoptosis biomarkers in human colorectal microadenomas. Cancer Epidemiol Biomarkers Prev 19: 351-357, 2010.

24. Livak KJ and Schmittgen TD: Analysis of relative gene expression data using real-time quantitative PCR and the 2(-Delta Delta C(T)) method. Methods 25: 402-408, 2001.

25. Li XY, Ota I, Yana I, Sabeh F and Weiss SJ: Molecular dissection of the structural machinery underlying the tissue-invasive activity of membrane type-1 matrix metalloproteinase. Mol Biol Cell 19: 3221-3233, 2008

26. Ougolkov AV, Yamashita K, Mai M and Minamoto T: Oncogenic beta-catenin and MMP-7 (matrilysin) cosegregate in late-stage clinical colon cancer. Gastroenterology 122: 60-71, 2002.

27. Bousserouel S, Kauntz H, Gossé F, Bouhadjar M, Soler L, Marescaux J and Raul F: Identification of gene expression profiles correlated to tumour progression in a preclinical model of colon carcinogenesis. Int J Oncol 36: 1485-1490, 2010.

28. Mauch S, Kolb C, Kolb B, Sadowski T and Sedlacek R: Matrix metalloproteinase-19 is expressed in myeloid cells in an adhesion-dependent manner and associates with the cell surface. J Immunol 168: 1244-1251, 2002.

29. Hieta N, Impola U, López-Otín C, Saarialho-Kere U and Kähäri VM: Matrix metalloproteinase-19 expression in dermal wounds and by fibroblasts in culture. J Invest Dermatol 121: 997-1004, 2003.

30. Kuperwasser C, Chavarria T, Wu M, Magrane G, Gray JW, Carey L, Richardson A and Weinberg RA: Reconstruction of functionally normal and malignant human breast tissues in mice. Proc Natl Acad Sci USA 101: 4966-4971, 2004.

31. Kalluri R: Basement membranes: structure, assembly and role in tumour angiogenesis. Nat Rev Cancer 3: 422-433, 2003.

32. Liotta LA and Kohn EC: The microenvironment of the tumourhost interface. Nature 411: 375-379, 2001.

33. Lynch CC and Matrisian LM: Matrix metalloproteinases in tumour-host cell communication. Differentiation 70: 561-573, 2002.

34. Ko K, Yazumi S, Yoshikawa K, Konda Y, Nakajima M, Chiba T and Takahashi R: Activation of fibroblast-derived matrix metalloproteinase-2 by colon-cancer cells in non-contact co-cultures. Int J Cancer 87: 165-171, 2000. 